Equi val ent compl ex conduct i vi ti es represent ing the effects of T-tubul es and fol ded surface menbranes on the el ectrical admittance and i medance of skel et al miscl es measured by ext er nal - el ect rode net hod

\begin{tabular}{|l|l|}
\hline 著者 & Seki ne Kat suhi sa \\
\hline 著者別表示 & 関根 克尚 \\
\hline $\begin{array}{l}\text { j our nal or } \\
\text { publ i cat i on ti t l e }\end{array}$ & Journal of Physi cs D: Appl i ed Physi cs \\
\hline vol une & 50 \\
\hline number & 50 \\
\hline page range & 505401 \\
\hline year & $2017-11-29$ \\
\hline URL & ht t p: //doi . or g/10. 24517/00050481 \\
\hline
\end{tabular}




\title{
Equivalent complex conductivities representing the effects of T-tubules and folded surface membranes on the electrical admittance and impedance of skeletal muscles measured by external-electrode method
}

\author{
Katsuhisa Sekine \\ Faculty of Health Sciences, Institute of Medical, Pharmaceutical and Health Sciences, \\ Kanazawa University, \\ 5-11-80 Kodatsuno, Kanazawa, Ishikawa 920-0942, Japan \\ E-mail: sekine@kenroku.kanazawa-u.ac.jp
}

\begin{abstract}
In order to represent the effects of T-tubules and folded surface membranes on the electrical admittance and impedance of skeletal muscles measured by the external-electrode method, analytical relations for the equivalent complex conductivities of hypothetical smooth surface membranes were derived. In the relations, the effects of each tubule were represented by the admittance of a straight cable. The effects of the folding of a surface membrane were represented by the increased area of surface membranes. The equivalent complex conductivities were represented as summation of these effects, and the effects of the T-tubules were different between the transversal and longitudinal directions. The validity of the equivalent complex conductivities was supported by the results of finite-difference method (FDM) calculations made using three-dimensional models in which T-tubules and folded surface membranes were represented explicitly. FDM calculations using the equivalent complex conductivities suggested that the electrically inhomogeneous structure due to the existence of muscle cells with T-tubules was sufficient for explaining the experimental results previously obtained using the external-electrode method. Results of FDM calculations in which the structural changes caused by muscle contractions were taken into account were consistent with reported experimental results.
\end{abstract}

Keywords:

bio-impedance, dielectric relaxation, electrical impedance myography, interfacial polarization, simulation

Classification numbers:

PACS

02.70.Bf Finite-difference methods

07.05.Tp Computer modeling and simulation

84.37.+q Measurements in electric variables (including voltage, current, resistance, capacitance,

87.19.Ff Muscles

87.19.rf Dielectric properties

87.19.xn Musculoskeletal diseases

87.64.-t Spectroscopic and microscopic techniques in biophysics and medical physics

87.64.Aa Computer simulation

Submitted to JOURNAL OF PHYSICS D: APPLIED PHYSICS 


\section{List of symbols in text}

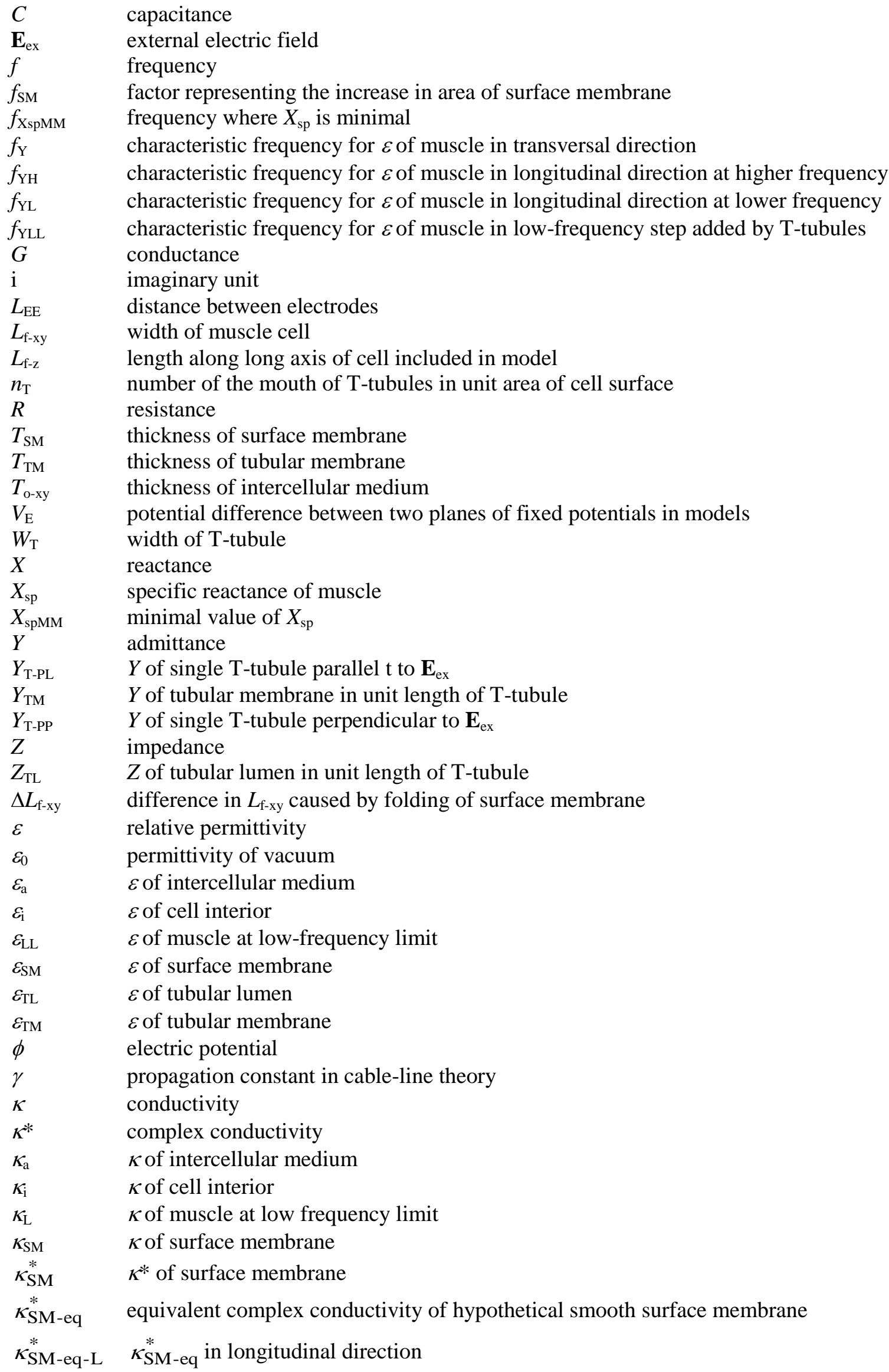




$\begin{array}{ll}\kappa_{\mathrm{SM}-\mathrm{eq}-\mathrm{T}}^{*} & \kappa_{\mathrm{SM}-\mathrm{eq}}^{*} \text { in transversal direction } \\ \kappa_{\mathrm{TL}}^{*} & \kappa \text { of tubular lumen } \\ \kappa_{\mathrm{TL}}^{*} & \kappa^{*} \text { of tubular lumen } \\ \kappa_{\mathrm{TM}} & \kappa \text { of tubular membrane } \\ \kappa_{\mathrm{TM}}^{*} & \kappa^{*} \text { of tubular membrane } \\ \rho & \text { resistivity of muscle } \\ \rho_{\mathrm{L}} & \rho \text { at low-frequency limit } \\ \omega & \text { angular frequency }\end{array}$

\section{Introduction}

A number of experimental investigations have demonstrated that measuring the electrical admittance, $Y$, and impedance, $Z$, of skeletal muscles is a promising noninvasive method for detecting the changes in muscles caused by contractions [1-3] and diseases [1, 4-8]. Despite these studies, a complete understanding of the physiological and histological changes in the muscles responsible for the observed changes in $Y$ and $Z$ has not yet been achieved, because the theoretical basis for data analysis is currently insufficient. In an earlier study [9], we attempted to analyze the observed data based on theoretical $Y$ and $Z$ evaluated numerically using the finite-difference method (FDM) in which three-dimensional models were used to represent the muscles. This study indicated that it is possible to estimate the physiological and histological origins of the observed changes in $Y$ and $Z$.

In the models used in our earlier study [9], individual muscle cells in a muscle were represented by a rectangular solid that was covered by a smooth membrane; we used them so that we could focus our efforts on studying both the electrically inhomogeneous structure of these cells, which consisted of an insulating surface membrane (sarcolemma), a conductive cell interior (sarcoplasm), and a conductive intercellular medium, and the histological structure of the muscles, in which these elongated muscle cells were closely packed in parallel. In the present study, this theoretical examination is extended so that the effects of T-tubules and the folding of the surface membranes of these cells could be investigated.

The contraction of muscles causes the distance between T-tubules to decrease due to the decrease in the sarcomere's length [10]. It also causes the surface membrane to make folding oriented perpendicular to the long axis of a muscle cell [11]. In other studies, the transmembrane capacitance of depolarized muscle cells was observed to increase due to the movement of charge between T-tubules and the sarcoplasmic reticulum of the cells $[12,13]$. Knowledge about the effects of such histological and physiological changes in T-tubules and surface membranes of cells on $Y$ and $Z$ of muscles is expected to provide the basis for the development of methods for analyzing the changes in $Y$ and $Z$ due to contractions [1-3].

The effects of T-tubules on the electrical properties of skeletal muscles have been investigated in single muscle cells using the intracellular electrode (IE) method [12-14] and in muscle tissues consisting of bundles of muscle cells using the extracellular electrode (EE) method $[1-8,15,16]$. In contrast to the detailed examinations of single muscle cells made using IE-methods [12-14], examinations of muscle tissues by EE-methods are considered to be limited. Initially, a circuit model consisting of a capacitor and a resistor (which were used to represent a tubular membrane and a tubular lumen, respectively) connected in series was proposed as a way to represent the effects of T-tubules [17]. However, an assessment of the validity of this model has not been carried out, because experimental difficulties due to the electrode polarization [18] have prevented a detailed comparison between theoretical and experimental results at low frequencies. This difficulty has led to a question [16] being asked about whether the theory of electrically inhomogeneous structures of muscle cells containing T-tubules adequately explains the experimental results obtained by EE-methods. In the present study, the effects of T-tubules were represented by analytical relations based on the cable-line theory [19]. Analytical relations for the equivalent complex conductivity, $\kappa_{\mathrm{SM} \text {-eq }}^{*}$, of a hypothetical 
smooth surface membrane were derived by considering the effects of T-tubules and folded surface membranes, and the validity of these relations was determined by comparing the results of FDM calculations made for two kinds of three dimensional models that mimicked EE-method measurements; in one model, referred to as the realistic model, the T-tubules and the folded surface membrane are represented explicitly, while in the other model, the smooth surface membrane is characterized by $\kappa_{\mathrm{SM} \text {-eq }}^{*}$.

In the FDM calculations made using the realistic model, the model was discretized into elements much smaller in size and much larger in number than in our previous study [9]; this was because the surface membrane in that study was modeled so as to replicate a smooth surface. This discretization increased the computational resources required and hindered the carrying out of calculations for large-sized realistic models. By using the equivalent complex conductivity, $\kappa_{\text {SM-eq }}^{*}$, we lightened the computational load and facilitated the calculation of large scale models; this meant that the results could be compared against those obtained for real muscle cells, and so the results from the FDM calculations made using $\kappa_{\text {SM-eq }}^{*}$ were compared with experimental results.

\section{Method}

\subsection{Realistic model}

2.1.1. Initial model developed for muscle cell with T-tubules and tissue. The initial model used had a similar structure to the one used in our previous study [9]. In the models, the long axis of the muscle cells was parallel to $z$-axis. Each cell was represented as a rectangular solid, whose cross section in $x y$-plane was a square of side length $L_{\mathrm{f}-\mathrm{xy}}$. In the muscle tissue, identically shaped cells were distributed in $x y$-plane at set intervals and were separated by an intercellular medium of uniform thickness, $T_{\text {o-xy. }}$ T-tubule networks were added to the cells in these models.

T-tubules are planer networks that are perpendicular to the long axis of muscle cells, and they consist of tubules that have diameters of 30-100 nm; these tubules surround myofibrils, which are about $1 \mu \mathrm{m}$ in diameter [10, 20]. In mammalian organisms, the T-tubules are located at the junction between the A and I bands [10]. Generally, the width of the A band corresponds to the length of a myosin filament, which is about $1.6 \mu \mathrm{m}$, whereas, the width of the I band changes from 2.4 to $0.4 \mu \mathrm{m}$ due to the change in the length of the sarcomere during muscle contractions from 4 to $2 \mu \mathrm{m}$ [10]. We constructed the T-tubules in our realistic model using this information.

Each tubule was represented by a rectangular solid whose cross section was a square of side length, $W_{\mathrm{T}}$. The T-tubule networks were constructed in $x y$-plane using tubules placed parallel to $x$ - and $y$-axes at intervals of $1 \mu \mathrm{m}$; this can been seen as mesh-A in figure 1 . In order to simplify initial models, the T-tubule networks were placed along $z$-axis at uniform intervals of $2 \mu \mathrm{m}$, as shown in figure 2(a).

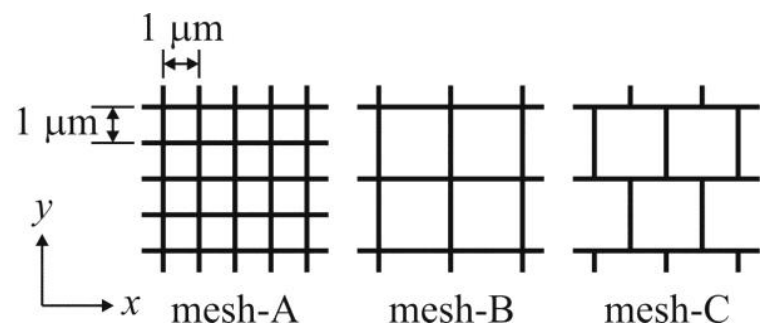

Figure 1. Shapes of T-tubule networks in the models. 


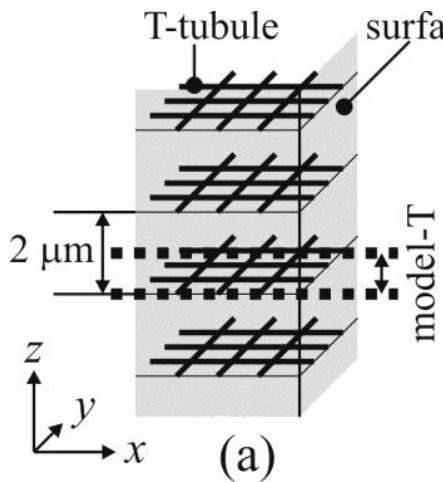

(a)

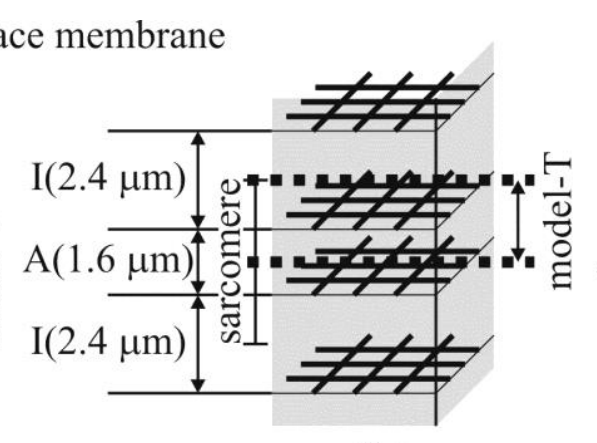

(b)

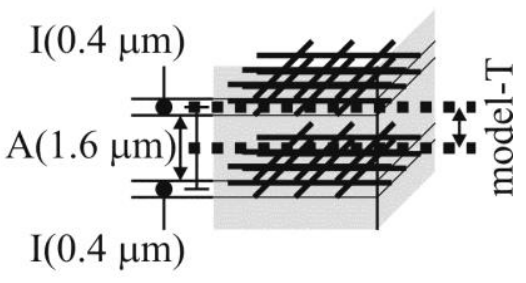

(c)

Figure 2. Positions of T-tubule networks along $z$-axis in (a) the initial cell model and the models corresponding to (b) the relaxed and (c) contracted states of a muscle. In (b), the widths of the A and I bands are $1.6 \mu \mathrm{m}$ and $2.4 \mu \mathrm{m}$, respectively. In (c), the width of the I band is $0.4 \mu \mathrm{m}$, while that of the A band is the same as that in (b).

Similarly to the earlier study we conducted [9], muscle tissues were represented by rectangular solids whose edges were parallel to $x$-, $y$ - and $z$-axes. FDM calculations were used to mimic two-terminal measurements made using electrodes placed on a surface parallel to $z x$-plane. In the measurements for the transversal direction, it was assumed that the electrodes occupied a region specified by $x \leq-L_{\mathrm{EE}} / 2$ and that specified by $x \geq L_{\mathrm{EE}} / 2$ on the surface, where $L_{\mathrm{EE}}$ was the distance between the electrodes. In the measurements for the longitudinal direction, the electrodes were assumed to occupy a region specified by $z \leq-L_{\mathrm{EE}} / 2$ and that specified by $z \geq L_{\mathrm{EE}} / 2$. The electric potential in the intercellular medium below the electrodes was assumed to be independent of depth. By taking into account the assumption described above about the potential caused by the electrodes and the symmetrical and periodic nature of the assumed muscle structure, rectangular-solid-shaped regions of minimum size were used in the FDM calculations.

Figures 3 and 4 show, Model-T, which was used to calculate $Y$ and $Z$ in the transversal direction, and Model-L, which was used to calculate them in the longitudinal direction, respectively. Model-T in figure 3 includes a structural unit, which was used to represent the assumed symmetrical and periodic structure of the muscles that are subjected to an external electric field, $\mathbf{E}_{\mathrm{ex}}$, which is parallel to $x$-axis. Model-T was surrounded by symmetric planes of $x=0$ and $y=0$ at the center of the muscle cell, $x=\left(L_{\mathrm{f}-\mathrm{xy}}+T_{\mathrm{o}-\mathrm{xy}}\right) / 2$ and $y=\left(L_{\mathrm{f}-\mathrm{xy}}+T_{\mathrm{o}-\mathrm{xy}}\right) / 2$ at the middle of the intercellular medium, $z=0$ at the central part between two neighboring T-tubule networks, and $z=1 \mu \mathrm{m}$ at the middle of one T-tubule network. In order to ensure that $\mathbf{E}_{\mathrm{ex}}$ was parallel to $x$-axis, the potentials, $\phi$, at $x=0$ and $x=$ $\left(L_{\mathrm{f}-\mathrm{xy}}+T_{\mathrm{o}-\mathrm{xy}}\right) / 2$ were fixed to be 0 and $V_{\mathrm{E}} / 2$, respectively; $V_{\mathrm{E}}$ in this model is the potential difference between two electrodes divided by the number of rows of cells between the electrodes. On the other planes, the normal component of the electric field was set to 0 . Model-L in figure 4 shows a quarter of a cell being surrounded by symmetric planes of $x=0, y=0, x=\left(L_{\text {f-xy }}+T_{\text {o-xy }}\right) / 2$ and $y=\left(L_{\text {f-xy }}+T_{\text {o-xy }}\right) / 2$. In this model, the region between two planes, $z=0$ at the central position between the two electrodes, and $z=L_{\mathrm{f}-\mathrm{z}}$ below one of the electrodes is included, where $L_{\mathrm{f}-\mathrm{z}}=L_{\mathrm{EE}} / 2$. $\phi$ was set to 0 at $z=0$, and was set to $V_{\mathrm{E}} / 2$ at $z=L_{\mathrm{f}-\mathrm{z}}$ in the region specified by $x \geq L_{\mathrm{f}-\mathrm{xy}} / 2$ or $y \geq L_{\mathrm{f}-\mathrm{xy}} / 2 ; V_{\mathrm{E}}$ in Model-L is the potential difference between two electrodes. On the other planes, the normal component of the electric field was set to 0 . 


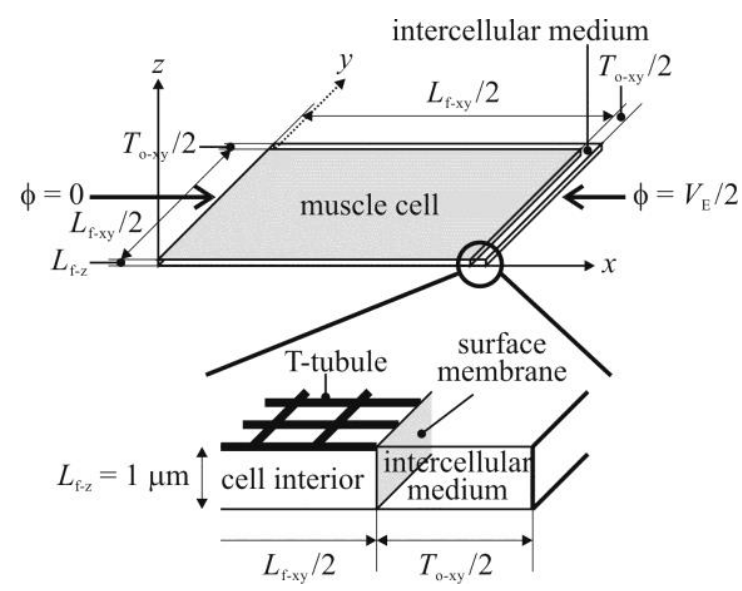

Figure 3. Initial structure of Model-T, which was used to calculate the admittance, $Y$, and the impedance, $Z$, in the transversal direction.

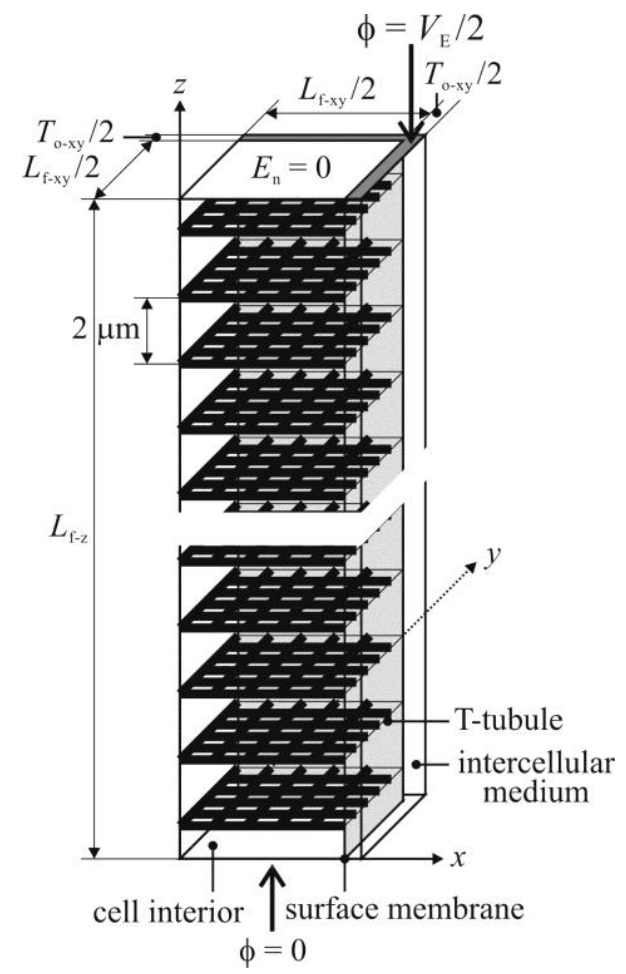

Figure 4. Initial structure of Model-L, which was used to calculate $Y$ and $Z$ in the longitudinal direction.

2.1.2. Parameter-values representing the structure of the muscle cells and tissues. In this subsection, we list the initial values used for the structural and electrical parameters of the models. The relative permittivity is represented by $\varepsilon$ and the conductivity by $\kappa$, and we differentiated parameters representing the cell interior, the intercellular medium, and the surface membrane using subscripts $i$, a, and SM, respectively. The initial values used were $\varepsilon_{\mathrm{i}}=\varepsilon_{\mathrm{a}}=80, \kappa_{\mathrm{i}}=\kappa_{\mathrm{a}}=2 \mathrm{~S} / \mathrm{m}, \varepsilon_{\mathrm{SM}}=10$ and $\kappa_{\mathrm{SM}}=0$. The thickness of the surface membrane, $T_{\mathrm{SM}}$, was set to $7 \mathrm{~nm}$. These values were the same as those used in our previous study [9]. With respect to the T-tubules, $W_{\mathrm{T}}=100 \mathrm{~nm}$ was used based on the structural information. Because the electrical properties unique to the membrane and lumen of T-tubules have not been experimentally observed [14], the relative permittivity and conductivity of the 
tubular lumen, $\varepsilon_{\mathrm{TL}}$ and $\kappa_{\mathrm{TL}}$, were set to the same values as those used for the cell interior and intercellular medium $\left(\varepsilon_{\mathrm{TL}}=80, \kappa_{\mathrm{TL}}=2 \mathrm{~S} / \mathrm{m}\right)$, while those values for the tubular membrane, $\varepsilon_{\mathrm{TM}}$ and $\kappa_{\mathrm{TM}}$ and the thickness of the tubular membrane, $T_{\mathrm{TM}}$, were the same as those used for the surface membrane $\left(\varepsilon_{\mathrm{TM}}=10, \kappa_{\mathrm{TM}}=0\right.$, and $\left.T_{\mathrm{TM}}=7 \mathrm{~nm}\right)$. In Model-T, $L_{\mathrm{f}-\mathrm{xy}}$ and $T_{\mathrm{o}-\mathrm{xy}}$ were set to $100 \mu \mathrm{m}$ and 5 $\mu \mathrm{m}$, respectively, similar to our previous study [9]. $L_{\mathrm{f}-\mathrm{xy}}, T_{\mathrm{o}-\mathrm{xy}}$ and $L_{\mathrm{f}-\mathrm{z}}$ in Model-L ( $L_{\mathrm{f}-\mathrm{xy}},=10 \mu \mathrm{m}, T_{\mathrm{o}-\mathrm{xy}}$ $=1 \mu \mathrm{m}$, and $L_{\mathrm{f}-\mathrm{z}}=200 \mu \mathrm{m}$ ) were smaller than those used in our previous study; this was due to the limitations imposed by the computational ability of our laboratory PCs.

The effects of the structural and electrical parameters on $Y$ and $Z$ were examined by changing $W_{\mathrm{T}}, \kappa_{\mathrm{TL}}, \varepsilon_{\mathrm{TM}}, \kappa_{\mathrm{TM}}, \varepsilon_{\mathrm{SM}}, \kappa_{\mathrm{SM}}, L_{\mathrm{f}-\mathrm{xy}}$ and $T_{\mathrm{o}-\mathrm{xy}}$. In Model-L, the effect of changing $L_{\mathrm{f}-\mathrm{z}}$ was also examined.

2.1.3. Examination of the effects caused by the mesh shape of T-tubule networks. The effects of the mesh shape of the T-tubule networks were examined using Model-T, shown in figure 3, and Model-L, shown in figure 4, in which the T-tubule networks were replaced by either mesh-B or -C, both of which are shown in figure 1. Because the shape of mesh-C is dependent on direction, additional FDM calculations for the transversal direction were carried out using a model similar to Model-T in figure 3 under the condition that $\mathbf{E}_{\text {ex }}$ is parallel to $y$-axis; this was produced using the boundary conditions of $\phi=0$ at $y=0$ and $\phi=V_{\mathrm{E}} / 2$ at $y=\left(L_{\mathrm{f}-\mathrm{xy}}+T_{\mathrm{o}-\mathrm{xy}}\right) / 2$.

2.1.4. Examination of the effects caused by the distance between T-tubule networks. The effect of the distance between the T-tubule networks changing due to muscle contractions was examined using the two models shown in figure 2(b) and 2(c), in which the sarcomere length was $4.0 \mu \mathrm{m}$ in the relaxed state (figure 2(b)) and $2.0 \mu \mathrm{m}$ in the contracted state (figure 2(c)). In these models, the width of the A band was set to $1.6 \mu \mathrm{m}$, and it was not varied. The width of the I band was set to $2.4 \mu \mathrm{m}$ in the relaxed state and $0.4 \mu \mathrm{m}$ in the contracted state. The T-tubule networks were placed at the junctions between the I and A bands.

The FDM calculations for the transversal direction were carried out using a model similar to Model-T. This model included a half of a sarcomere bounded by two planes, and these are represented by the bold dotted lines in figures 2(b) and 2(c); they are located at the centers of the neighboring I and A bands. As such, $L_{\mathrm{f}-\mathrm{z}}=2.0 \mu \mathrm{m}$ and $L_{\mathrm{f}-\mathrm{z}}=1.0 \mu \mathrm{m}$ in the relaxed and contracted states, respectively, in Model-T. The FDM calculations for the longitudinal direction were carried out using a model similar to Model-L in figure 4, which included 25 sarcomeres. As such, $L_{\mathrm{f}-\mathrm{z}}=100 \mu \mathrm{m}$ and $L_{\mathrm{f}-\mathrm{z}}=50 \mu \mathrm{m}$ in the relaxed and contracted states, respectively, in this model.

2.1.5. Examination of the effects of folding of surface membranes. The effects of the folding of surface membranes were examined using models that had structures similar to Model-T and Model-L, except that they did not have T-tubules. Figure 5 shows the cross-section of these models in planes parallel to $y z$ - or $z x$-plane. The folding of a surface membrane oriented perpendicularly to the long axis of a muscle cell was represented by a change in $L_{\mathrm{f}-\mathrm{xy}}$ with $z$ in the shape of a square wave that had a wavelength of $1 \mu \mathrm{m}$. The effects of the amplitude of the square wave, which is given by $\Delta L_{\mathrm{f}-\mathrm{xy}} / 2$, were examined using two kinds of models: one in which $\Delta L_{\mathrm{f}-\mathrm{xy}} / 2=0.1 \mu \mathrm{m}$ and one in which $\Delta L_{\mathrm{f}-\mathrm{xy}} / 2=0.2$ $\mu \mathrm{m}$. In the models for both the transversal and the longitudinal directions, $L_{\mathrm{f}-\mathrm{xy}}=100 \mu \mathrm{m}$ and $T_{\mathrm{o}-\mathrm{xy}}=5$ $\mu \mathrm{m}$. $L_{\mathrm{f}-\mathrm{z}}$ was set to $1 \mu \mathrm{m}$ and $500 \mu \mathrm{m}$ in the models for the transversal and longitudinal directions, respectively. 


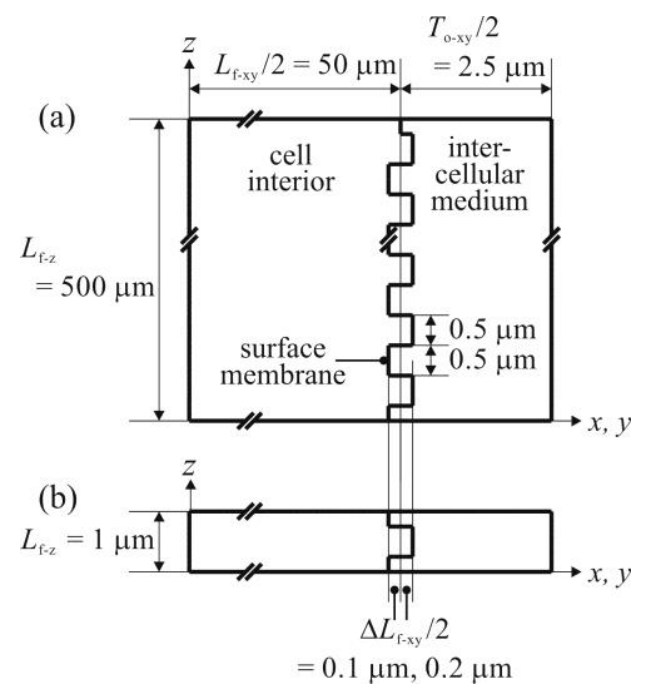

Figure 5. Cross-section of the models in planes parallel to $y z$ - or $z x$-plane; these models were used to examine the effects of the folding of surface membranes on $Y$ and $Z$ in (a) the longitudinal and (b) the transversal directions.

\subsubsection{Modified models used to examine effects of the direction of the external fields in the} transversal direction. As will be described in 2.2., the equivalent complex permittivity $\kappa_{\mathrm{SM} \text {-eq-T }}^{*}$ in the transversal direction was derived under the assumption that cells consisted of T-tubules and surface membranes both parallel or perpendicular to $\mathbf{E}_{\mathrm{ex}}$. In order to examine the utility of this equivalent complex conductivity in cases where the angle between a T-tubule and $\mathbf{E}_{\mathrm{ex}}$ and that between a surface membrane and $\mathbf{E}_{\text {ex }}$ deviated from the assumed ones, FDM calculations were carried out using the models shown in figures 6 and 7 instead of the model shown in figure 3 . $\mathbf{E}_{\mathrm{ex}}$ crosses the T-tubules and surface membranes at an angle of 45 degrees in figure 6 , and is nonuniform in figure 7. In the model in figure $6, L_{\mathrm{f}-\mathrm{xy}}=10 \mu \mathrm{m}$ and $T_{\mathrm{o}-\mathrm{xy}}=1 \mu \mathrm{m}$. In the model in figure $7, L_{\mathrm{f}-\mathrm{xy}}=8 \mu \mathrm{m}$ and $T_{\mathrm{o}-\mathrm{xy}}$ $=2 \mu \mathrm{m}$. In both models, the structure of the muscle cells along $z$-axis is the same as that shown in figure 3.

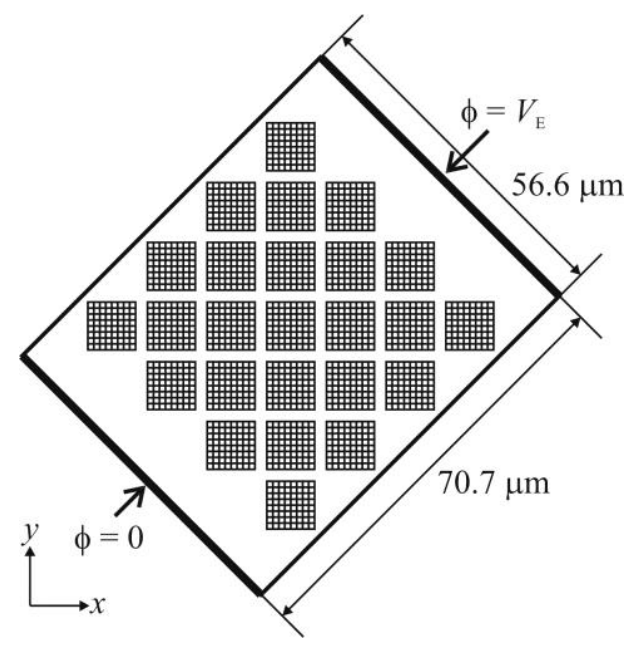

Figure 6. The model used to calculate $Y$ and $Z$ in the transversal direction under an external electric field, $\mathbf{E}_{\mathrm{ex}}$, that crossed the T-tubules and surface membranes at an angle of 45 degrees. 


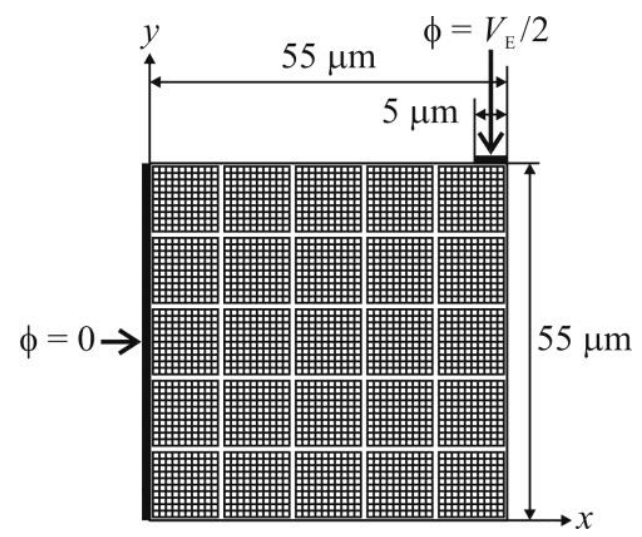

Figure 7. The model used to calculate $Y$ and $Z$ in the transversal direction under nonuniform $\mathbf{E}_{\text {ex }}$.

\subsection{Equivalent complex conductivity of hypothetical smooth surface membrane}

In order to represent the effects of the T-tubules and the folding of surface membranes, analytical relations for the equivalent complex conductivity of hypothetical smooth surface membranes were derived under the following assumptions:

(a) The admittance of each tubule can be obtained for a straight cable without branching by using the cable-line theory [19].

(b) The electric potential in the cell interior is 0 .

(c) As shown in figure 8, the T-tubule networks consisted of tubules parallel (T-PL) and perpendicular (T-PP) to $\mathbf{E}_{\mathrm{ex}}$.

(d) The muscle cells consisted of cell surfaces parallel (CS-PL) and perpendicular (CS-PP) to $\mathbf{E}_{\text {ex }}$.

(e) The sum of the admittances of the tubules and surface membranes contributes to the values of $Y$ and $Z$ of the muscles.

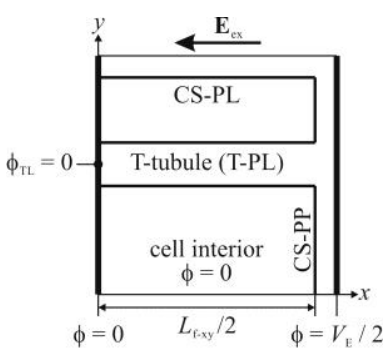

(T-1)

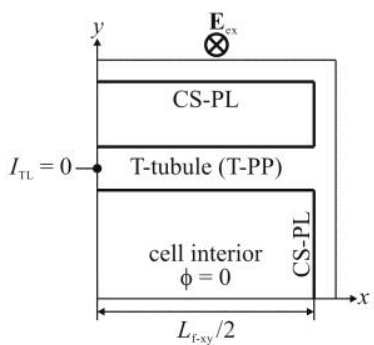

(L-1)

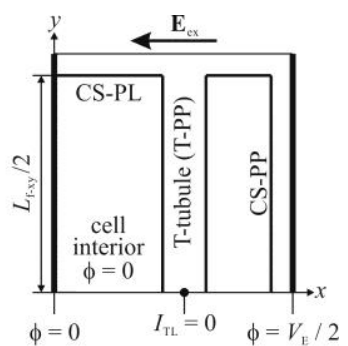

(T-2)

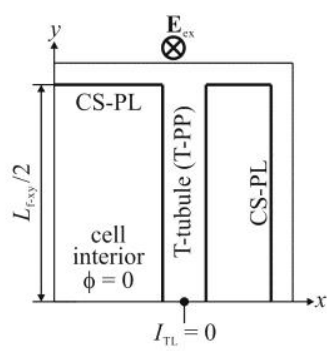

(L-2)

Figure 8. Classification of the tubules and cell surfaces in Model-T (T-1 and T-2) and in Model-L (L-1 and L-2). 
As described in Appendix A, the equivalent complex conductivity in the transversal direction, $\kappa_{\mathrm{SM}-\mathrm{eq}-\mathrm{T}}^{*}$, and in the longitudinal direction, $\kappa_{\mathrm{SM}-\mathrm{eq}-\mathrm{L}}^{*}$, can be represented by the following relations:

$\kappa_{\mathrm{SM}-\mathrm{eq}-\mathrm{T}}^{*}=\kappa_{\mathrm{SM}}^{*} f_{\mathrm{SM}}+T_{\mathrm{SM}} n_{\mathrm{T}}\left(Y_{\mathrm{T}-\mathrm{PP}}+3 Y_{\mathrm{T}-\mathrm{PL}}\right) / 4$

$\kappa_{\mathrm{SM}-\mathrm{eq}-\mathrm{L}}^{*}=\kappa_{\mathrm{SM}}^{*} f_{\mathrm{SM}}+T_{\mathrm{SM}} n_{\mathrm{T}} Y_{\mathrm{T}-\mathrm{PP}}$

where $\kappa_{\mathrm{SM}}^{*}$ is the complex conductivity of a surface membrane, $f_{\mathrm{SM}}$ is a factor representing the increase in the area of a surface membrane due to folding, $T_{\mathrm{SM}}$ is the thickness of a surface membrane, and $n_{\mathrm{T}}$ is the number of mouths of the tubules in unit area of a hypothetical smooth surface membrane. The complex conductivities were given by relations in the form of $\kappa^{*}=\kappa+\mathrm{i} \omega \varepsilon \varepsilon_{0}$, where $\mathrm{i}$ is the imaginary unit, $\omega$ is angular frequency (represented by $\omega=2 \pi f$, where $f$ represents frequency), and $\varepsilon_{0}$ is the permittivity of vacuum. The term $Y_{\mathrm{T}-\mathrm{PP}}$ represents the admittance of a single tubule perpendicular to $\mathbf{E}_{\mathrm{ex}}$, and $Y_{\mathrm{T}-\mathrm{PL}}$ represents that of a single tubule parallel to $\mathbf{E}_{\mathrm{ex}}$. These two terms can be expressed as the following relations:

$$
\begin{aligned}
& Y_{\mathrm{T}-\mathrm{PP}}=\sqrt{\frac{Y_{\mathrm{TM}}}{Z_{\mathrm{TL}}}} \times \frac{1-\exp \left(-\gamma L_{\mathrm{f}-\mathrm{xy}}\right)}{1+\exp \left(-\gamma L_{\mathrm{f}-\mathrm{xy}}\right)}, \\
& Y_{\mathrm{T}-\mathrm{PL}}=\sqrt{\frac{Y_{\mathrm{TM}}}{Z_{\mathrm{TL}}}} \times \frac{1+\exp \left(-\gamma_{\mathrm{f}-\mathrm{xy}}\right)}{1-\exp \left(-\gamma L_{\mathrm{f}-\mathrm{xy}}\right)},
\end{aligned}
$$

where $Y_{\mathrm{TM}}$ and $Z_{\mathrm{TL}}$ are the admittance of a tubular membrane and the impedance of a tubular lumen, respectively, in unit length, and $\gamma=\sqrt{Y_{\mathrm{TM}} Z_{\mathrm{TL}}}$. In the models used in the present study, $Y_{\mathrm{TM}}=4 W_{\mathrm{T}} \kappa_{\mathrm{TM}}^{*}$ and $Z_{\mathrm{TL}}=1 /\left(W_{\mathrm{T}}^{2} \kappa_{\mathrm{TL}}^{*}\right)$, where $\kappa_{\mathrm{TM}}^{*}$ and $\kappa_{\mathrm{TL}}^{*}$ are the complex conductivities of a tubular membrane and lumen, respectively.

\subsection{FDM calculations}

The FDM calculations were carried out using a method similar to the one developed by Asami [21] using the realistic models described in 2.1. and the models consisting of muscle cells whose surface membranes are smooth and characterized by $\kappa_{\mathrm{SM}-\mathrm{eq}-\mathrm{T}}^{*}$ or $\kappa_{\mathrm{SM}-\mathrm{eq}-\mathrm{L}}^{*}$.

The current that passed through the surfaces of the fixed potentials was obtained using the potential distribution obtained from the FDM calculations. $Y$ and $Z$ were evaluated from the current and the potential difference, and were represented using capacitance, $C$, conductance, $G$, resistance, $R$, and reactance, $X$; these are related to $Y$ and $Z$ as $Y=G+\mathrm{i} \omega C$ and $Z=R+\mathrm{i} X$. In some cases, $\varepsilon, \kappa, \rho$ (resistivity), and $X_{\text {sp }}$ (specific reactance) were evaluated from $C, G, R$, and $X$, respectively, using the area of the surfaces of the fixed potentials and the distance between them. 


\section{Results and discussion}

\subsection{Assessment of the validity of the equivalent complex conductivities}

The validity of the equivalent complex conductivities, $\kappa_{\mathrm{SM} \text {-eq-T }}^{*}$ and $\kappa_{\mathrm{SM}-\mathrm{eq}-\mathrm{L}}^{*}$, was assessed by comparing the results obtained by FDM calculations using the realistic model and calculations using the model with a smooth surface membrane, which is characterized by $\kappa_{\text {SM-eq-T }}^{*}$ and $\kappa_{\text {SM-eq-L }}^{*}$. Aside from the changes noted, the realistic model had the structure described in 2.1.1. and the initial parameter values described in 2.1.2. The equivalent complex conductivities corresponding to the realistic models were evaluated by the method described in 2.2 ..

3.1.1. Choice of equivalent complex conductivities dependent on direction. Figures 9 and 10 show the results of the FDM calculations using the models that did not contain the T-tubules, the realistic model in which the T-tubules were represented, and the models that characterized surface membranes using $\kappa_{\text {SM-eq-T }}^{*}$ and $\kappa_{\text {SM-eq-L }}^{*}$. As seen from these figures, the T-tubules caused an increase in $C$ at low frequencies in both the transversal and the longitudinal directions (figures 9 (a) and 10(a), respectively). The T-tubules made the frequency-dependence of $G$ (figures 9 (a) and 10(a)), $R$, and $X$ (figures 9(b) and 10(b)) more gradual than when no T-tubules were contained. The frequencies at which $R$ decreased with frequency and $X$ was minimal were found to decrease because of the T-tubules.

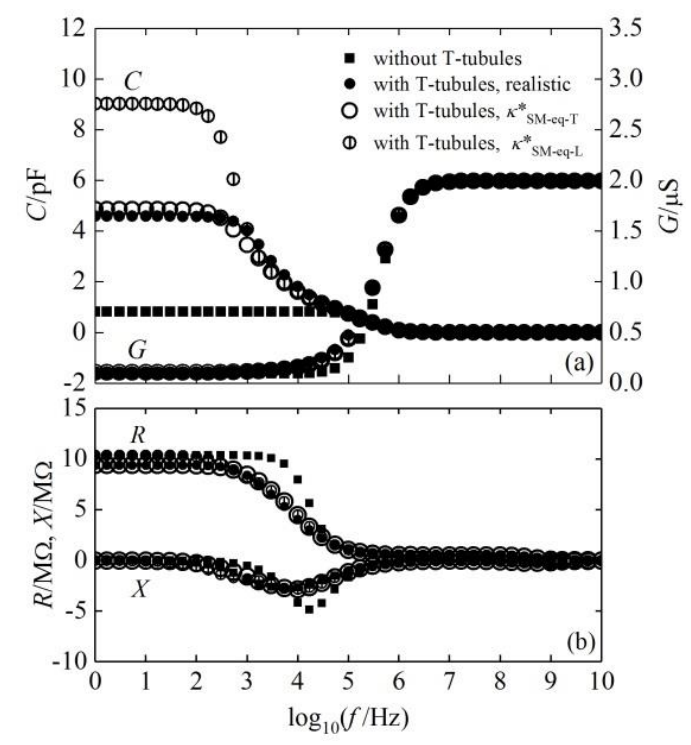

Figure 9. Frequency-dependence of $C, G, R$, and $X$ in the transversal direction, as obtained by FDM calculations in which a cell model did not contain any T-tubules, a realistic model in which T-tubules were represented explicitly, and models in which smooth surface membranes were characterized by the equivalent complex conductivities $\kappa_{\mathrm{SM} \text {-eq-T }}^{*}$ and $\kappa_{\mathrm{SM} \text {-eq-L }}^{*}$ in the transversal direction and longitudinal directions. 


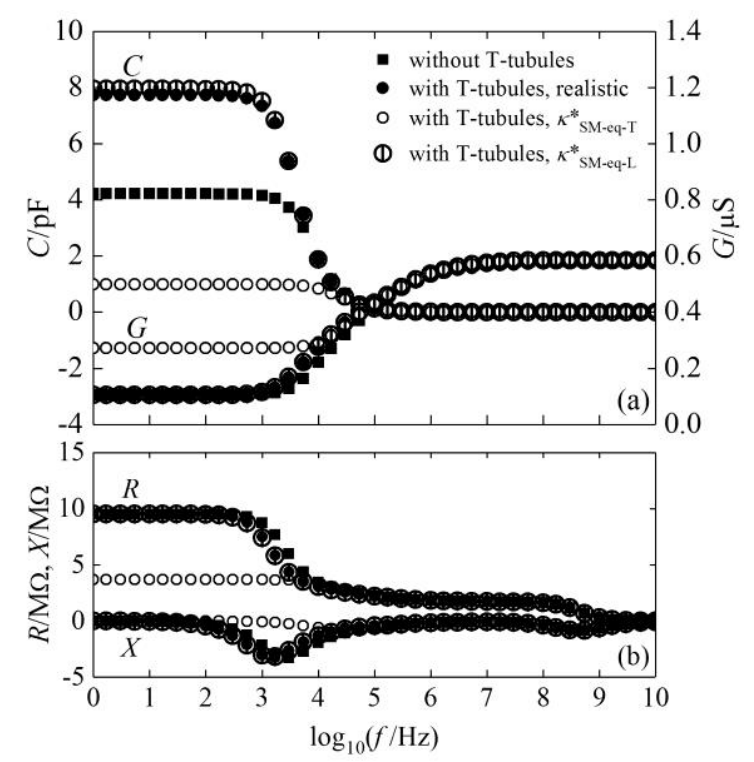

Figure 10. Frequency-dependence of $C, G, R$, and $X$ in the longitudinal direction, as obtained by FDM calculations in which a cell model did not contain any T-tubules, a realistic model containing T-tubules, and models with smooth surface membranes characterized by $\kappa_{\mathrm{SM} \text {-eq-T }}^{*}$ and $\kappa_{\mathrm{SM} \text {-eq-L }}^{*}$.

As can be seen from figures 9 and 10, the effects of the T-tubules were successfully recreated using $\kappa_{\mathrm{SM} \text {-eq-T }}^{*}$ and $\kappa_{\mathrm{SM} \text {-eq-L }}^{*}$, if the choice was suitable. At low frequencies, the values of $C$ evaluated using $\kappa_{\text {SM-eq-T }}^{*}$ and $\kappa_{\text {SM-eq-L }}^{*}$ were slightly larger than those obtained from the realistic model in both the transversal and the longitudinal directions. These deviations can be attributable to the area of the tubular membrane being overestimated by $\kappa_{\text {SM-eq-T }}^{*}$ and $\kappa_{\text {SM-eq-L }}^{*}$, in which the decrease in the area of the tubular membranes due to the crossing of the tubules is disregarded. Bad choice from the equivalent complex conductivities caused significant deviations at low frequencies in $C$ in the transversal direction (figure $9(\mathrm{a})$ ) and in $C, G, R$, and $X$ in the longitudinal direction (figure $10)$.

3.1.2. Effects of the parameter-values representing the structure of muscle cells and tissue. In the examination of the effects of changes in the parameter-values described in 2.1.2., $\kappa_{\mathrm{SM} \text {-eq-T }}^{*}$ and $\kappa_{\mathrm{SM}-\mathrm{eq}-\mathrm{L}}^{*}$ were found to reasonably represent the changes in $C, G, R$, and $X$ evaluated using the realistic model, although the data are not shown in this study. This supports the validity of using $\kappa_{\mathrm{SM} \text {-eq-T }}^{*}$ and $\kappa_{\mathrm{SM} \text {-eq-L }}^{*}$ for this purpose. The success of the use of $\kappa_{\mathrm{SM}-\mathrm{eq}-\mathrm{L}}^{*}$ at various values of $L_{\mathrm{f}-\mathrm{xy}}, T_{\mathrm{o}-\mathrm{xy}}$, and $L_{\mathrm{z}}$ in the longitudinal direction particularly supports our finding with regard to the validity of using FDM calculations for large cell models with smooth surface membranes characterized by $\kappa_{\mathrm{SM}-\mathrm{eq}-\mathrm{L}}^{*}$.

3.1.3. Effects of the mesh shape of the T-tubule network. Figure 11 shows the frequency-dependence of $C, G, R$, and $X$ in the transversal direction as obtained by the FDM calculations described in 2.1.3.. In the realistic model, the results of FDM calculations obtained using mesh-C were independent of the direction of $\mathbf{E}_{\mathrm{ex}}$, although the tubules in mesh-C were out of alignment in $y$-direction. The results of the calculations using meshes - $\mathrm{B}$ and $-\mathrm{C}$ agreed with each other. Furthermore, in the longitudinal direction, the results of the FDM calculations were independent 
of the mesh-shape (data not shown). In both the transversal and the longitudinal directions, the results of FDM calculations using the models with smooth surface membranes characterized by $\kappa_{\text {SM-eq-T }}^{*}$ and $\kappa_{\mathrm{SM} \text {-eq-L }}^{*}$ agreed with those obtained using the realistic models with meshes -B and -C. These results support the validity of the assumption that the admittance of a tubule can be represented by that of a straight cable.

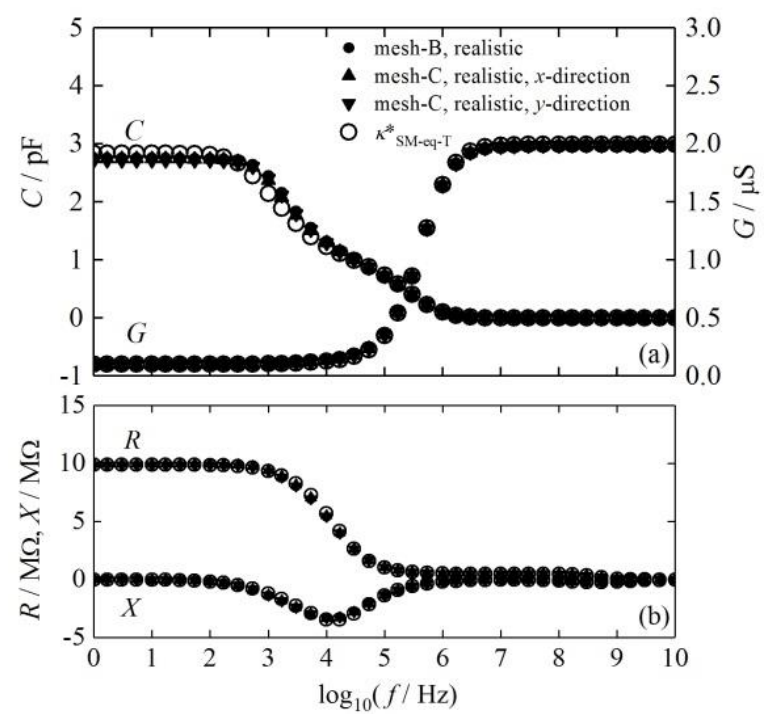

Figure 11. Frequency-dependence of $C, G, R$, and $X$ in the transversal direction as obtained by FDM calculations using a model with T-tubules in the shape specified by mesh-B and that of a model with T-tubules in the shape specified by mesh-C under $\mathbf{E}_{\mathrm{ex}}$ in $x$ - and $y$-directions, and that of a model with a smooth surface membrane characterized by $\kappa_{\mathrm{SM}-\mathrm{eq}-\mathrm{T}}^{*}$.

3.1.4. Effect of a non-uniform distance between T-tubule networks. Figure 12 shows the frequency-dependence of $C, G, R$, and $X$ in the transversal direction as obtained by the FDM calculations described in 2.1.4.. When compared with figure 9 , in which there is a uniform distance between T-tubule networks, the values of $C$ below $100 \mathrm{~Hz}$ at sarcomere lengths of $2 \mu \mathrm{m}$ and $4 \mu \mathrm{m}$ and the values of $G$ above $1 \mathrm{MHz}$ at a sarcomere length of $4 \mu \mathrm{m}$, shown in figure 12, were larger than those shown in figure 9 . The values of $R$ below $100 \mathrm{~Hz}$ at a sarcomere length of $4 \mu \mathrm{m}$, shown in figure 12 , were smaller than those shown in figure 9 . These differences can be explained by the differences in the areas of the tubular membranes and $L_{\mathrm{f}-\mathrm{z}}$ in the models used in the calculations. The equivalent complex conductivities were found to be suitable for representing the effects of the T-tubules, irrespective of whether the distance between T-tubule networks is not uniform; however, results in the longitudinal direction are not shown. This supports the validity of the assumption that the summation of the admittance of the tubules contributes to $Y$ and $Z$ of muscles. 


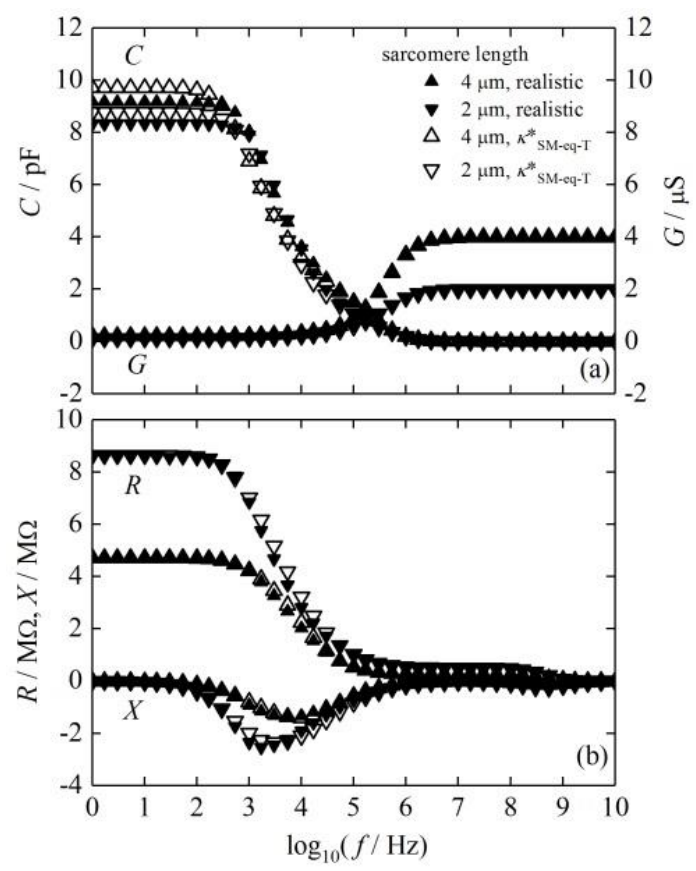

Figure 12. Frequency-dependence of $C, G, R$, and $X$ in the transversal direction as obtained by FDM calculations using the realistic model in which lengths of the sarcomeres were $4 \mu \mathrm{m}$ (figure 2(b)) and 2 $\mu \mathrm{m}$ (figure 2(c)), and a model in which with the smooth surface membrane was characterized by $\kappa_{\text {SM-eq-T }}^{*}$.

3.1.5. Effects of folding of surface membrane. Figure 13 shows the frequency-dependence of $C, G$, $R$, and $X$ in the transversal direction, as obtained by the FDM calculations described in 2.1.5. The folding caused the increase in $C$ below $100 \mathrm{kHz}$, the decrease in the frequency-region in which $R$ decreased with frequency, and the decrease in the frequency at which $X$ was minimum. The equivalent complex conductivities were found to be suitable for representing the effects of the folding of surface membranes, although the results in the longitudinal direction are not shown.

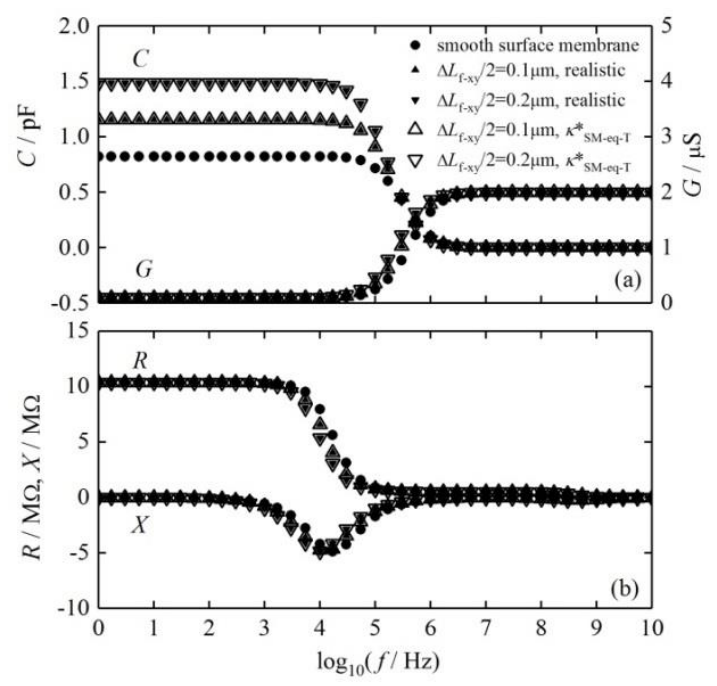

Figure 13. Frequency-dependence of $C, G, R$, and $X$ in the transversal direction as obtained by FDM calculations using a model containing a non-folded smooth membrane, the realistic model with a folded surface membrane, and a model with a smooth surface membrane characterized by $\kappa_{\text {SM-eq-T }}^{*}$. $\Delta L_{\mathrm{f}-\mathrm{xy}} / 2$ is the amplitude of the square wave used to represent the folding of the surface membrane. 
3.1.6. Effects of the direction of the external fields in the transversal direction. Figures 14 and 15 show the frequency-dependence of $C, G, R$, and $X$ in the transversal direction, as obtained by FDM calculations using the models shown in figures 6 and 7, respectively, and as described in 2.1.6.. In figures 14 and 15, the increase in $C$ as the frequency decreases is due to the T-tubules, and it took place in a frequency-region above that shown in figure 9. The ratio of the amount of $C$ around 100 $\mathrm{kHz}$ increased by the existence of the T-tubules to that around $1 \mathrm{MHz}$ increased by the existence of surface membrane was smaller than that shown in figure 9. The existence of the T-tubules did not cause noticeable changes in $G, R$, and $X$. Regardless of these differences, $\kappa_{\mathrm{SM} \text {-eq-T }}^{*}$ was successful in representing the effects of the T-tubules. As described in 2.2., $\kappa_{\mathrm{SM} \text {-eq-T }}^{*}$ was derived under the assumption that the muscle cells consisted of tubules and surface membranes that were parallel or perpendicular to $\mathbf{E}_{\mathrm{ex}}$. The results described above demonstrate that $\kappa_{\mathrm{SM} \text {-eq-T }}^{*}$ can be utilized in cases in which the angle between the tubules and $\mathbf{E}_{\mathrm{ex}}$ and that between the surface membranes and $\mathbf{E}_{\mathrm{ex}}$ deviate from the assumed ones.

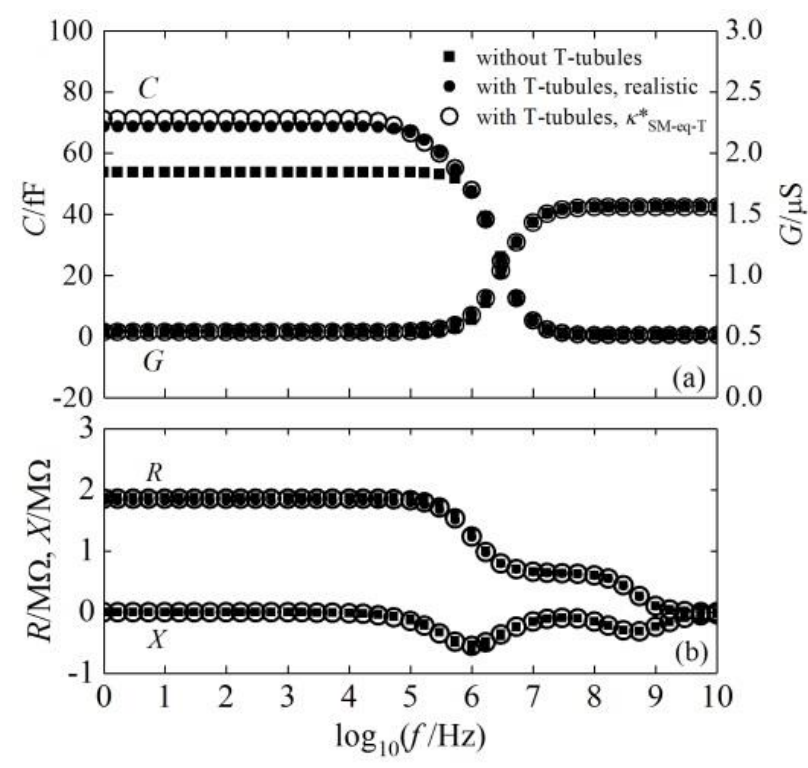

Figure 14. Frequency-dependence of $C, G, R$, and $X$ in the transversal direction as obtained by the FDM calculations using the model shown in figure 6, in which $\mathbf{E}_{\mathrm{ex}}$ crosses the T-tubules and the surface membranes at an angle of 45 degrees. The results of the calculations using a model without T-tubules, a realistic cell model containing T-tubules, and a model with a smooth surface membrane characterized by $\kappa_{\mathrm{SM}-\mathrm{eq}-\mathrm{T}}^{*}$ are all shown in the figure. 


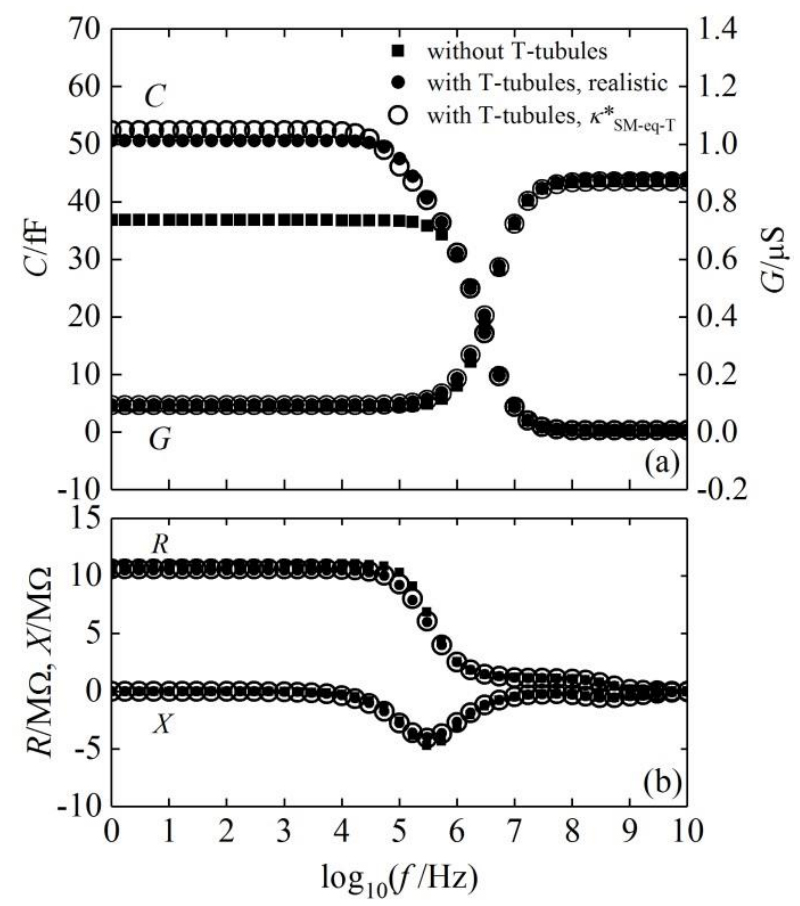

Figure 15. Frequency-dependence of $C, G, R$, and $X$ in the transversal direction as obtained by FDM calculations using the model shown in figure 7, in which $\mathbf{E}_{\mathrm{ex}}$ is non-uniform. The results of the calculations using a model without T-tubules, a realistic cell model containing T-tubules, and a model with a smooth surface membrane characterized by $\kappa_{\mathrm{SM} \text {-eq-T }}^{*}$ are shown in the figure.

\subsection{Effects of T-tubules in models large enough to be comparable to real muscle cells}

The use of the equivalent complex conductivities made it possible to examine the effects of T-tubules in a longitudinal direction using models that were large enough to be comparable to real muscle cells. Figure 16 shows the frequency-dependence of $\varepsilon, \kappa, \rho$, and $X_{\mathrm{sp}}$ in the transversal and longitudinal directions calculated using models similar to those shown in figures 3 and 4; these models had smooth surface membranes that were characterized by $\kappa_{\mathrm{SM}-\mathrm{eq}-\mathrm{T}}^{*}$ and $\kappa_{\mathrm{SM}-\mathrm{eq}-\mathrm{L}}^{*}$. Similarly to our previous study [9], $L_{\mathrm{f}-\mathrm{xy}}$ and $T_{\mathrm{o}-\mathrm{xy}}$ were set to $100 \mu \mathrm{m}$ and $5 \mu \mathrm{m}$, respectively, in models for both directions, while $L_{\mathrm{f}-\mathrm{z}}$ in the model for the transversal and longitudinal directions was set to $1 \mu \mathrm{m}$ and $1 \mathrm{~mm}$, respectively. The equivalent complex conductivities were evaluated using the initial parameter-values described in 2.1.2., mesh-A (shown in figure 1) and the distribution of T-tubules along $z$-axis at uniform intervals of $2 \mu \mathrm{m}$ (shown in figure 2(a)). 

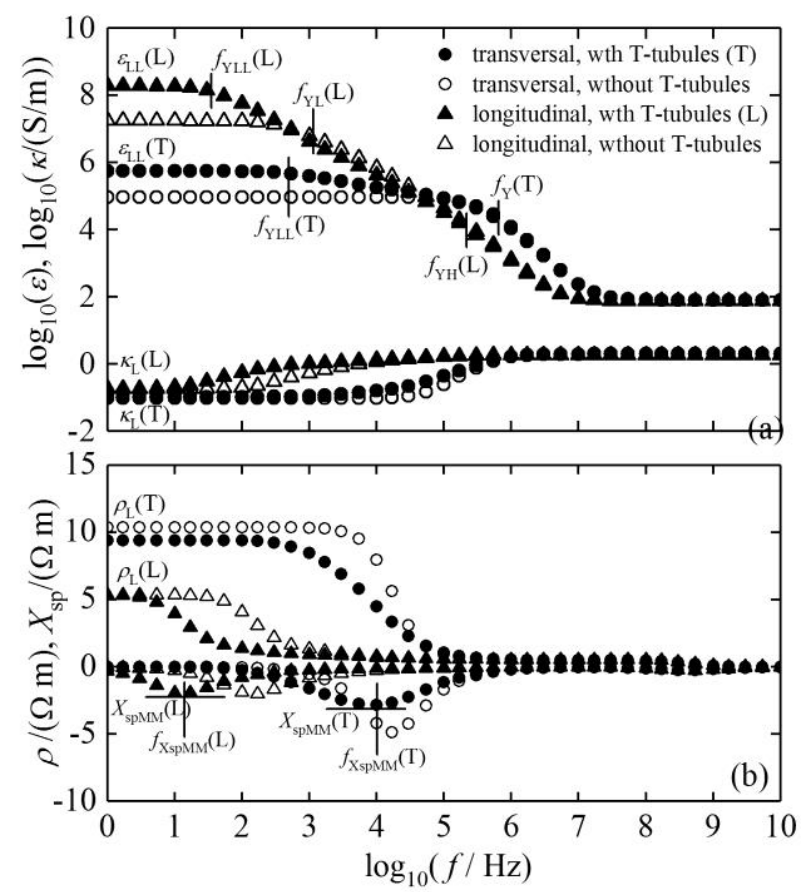

Figure 16. Frequency-dependence of $\varepsilon, \kappa, \rho$, and $X_{\mathrm{sp}}$ in the transversal and longitudinal directions; these values were obtained using models large enough to be comparable to real muscle cells, and smooth surface membranes characterized by $\kappa_{\mathrm{SM} \text {-eq-T }}^{*}$ or $\kappa_{\mathrm{SM} \text {-eq-L }}^{*} \cdot \varepsilon, \kappa, \rho$, and $X_{\mathrm{sp}}$ of the model that does not contain any T-tubules are also included.

As can be seen in figure 16, the T-tubules caused an additional step in the frequency-dependence of $\varepsilon$ around $1 \mathrm{kHz}$ in the transversal direction and that around $30 \mathrm{~Hz}$ in the longitudinal direction. The T-tubules also caused frequency-region in which $\kappa, \rho$, and $X_{\mathrm{sp}}$ change with frequency to decrease.

Table 1 shows the effects of the parameter-values related to the T-tubules on the frequency-dependence of $\varepsilon, \kappa, \rho$, and $X_{\mathrm{sp}}$. Because the T-tubules caused an additional step in $\varepsilon$ at low frequencies, the effects on $\varepsilon$ were represented using $\varepsilon_{\mathrm{LL}}$ at the low-frequency limit and the characteristic frequency, $f_{\mathrm{YLL}}$, of the additional step in addition to the characteristic frequency, $f_{\mathrm{Y}}$, in the transversal direction and $f_{\mathrm{YL}}$ and $f_{\mathrm{YH}}$ in the longitudinal direction; these parameters were used in the previous study [9] to represent the frequency-dependence of $\varepsilon . \kappa_{\mathrm{L}}$ and $\rho_{\mathrm{L}}$ are the values of $\kappa$ and $\rho$, respectively, at the low-frequency limit. $X_{\mathrm{spMM}}$ was the minimum value of $X_{\mathrm{sp}}$, and $f_{\mathrm{XspMM}}$ was the frequency at which $X_{\mathrm{sp}}=X_{\mathrm{spMM}}$. 
Table 1. Effects of the increase in the parameter-values used to characterize the structure of the T-tubules on the frequency-dependence of $\varepsilon, \kappa, \rho$, and $X_{\mathrm{sp}}$ in the transversal and longitudinal directions, where $\uparrow, \downarrow$ and - represent the notion that an increase in the parameter-values characterizing the structure of the T-tubules caused an increase, decrease, and no effect, respectively, on these particular parameters.

\begin{tabular}{lcccccc} 
& $n_{\mathrm{T}}$ & $W_{\mathrm{T}}$ & $\kappa_{\mathrm{TL}}{ }^{\mathrm{a}}$ & $\kappa_{\mathrm{TL}}{ }^{\mathrm{b}}$ & $\varepsilon_{\mathrm{TM}}$ & $\kappa_{\mathrm{TM}}$ \\
\hline \multicolumn{2}{l}{ Transversal direction } & & & & & \\
$\varepsilon_{\mathrm{LL}}$ & $\uparrow$ & $\uparrow$ & - & $\downarrow$ & $\uparrow$ & $\downarrow$ \\
$f_{\mathrm{YLL}}$ & - & $\uparrow$ & $\uparrow$ & $\uparrow$ & $\downarrow$ & - \\
$f_{\mathrm{Y}}$ & - & - & - & $\downarrow$ & - & - \\
$\kappa_{\mathrm{L}}$ & $\uparrow$ & $\uparrow$ & $\uparrow$ & $\uparrow$ & - & $\uparrow$ \\
$\rho_{\mathrm{L}}$ & $\downarrow$ & $\downarrow$ & $\downarrow$ & $\downarrow$ & - & $\downarrow$ \\
$X_{\mathrm{spMM}}$ & $\uparrow$ & $\uparrow$ & $\uparrow$ & $\uparrow$ & $\uparrow$ & - \\
$f_{\mathrm{XspMM}}$ & $\downarrow$ & $\downarrow$ & - & - & - & -
\end{tabular}

Longitudinal direction

$\begin{array}{lcccccc}\varepsilon_{\mathrm{LL}} & \uparrow & \uparrow & - & - & \uparrow & \downarrow \\ f_{\mathrm{YLL}} & - & \downarrow & \uparrow & - & \downarrow & \uparrow \\ f_{\mathrm{YL}} & - & - & - & - & - & - \\ f_{\mathrm{YH}} & - & - & - & \downarrow & - & - \\ \kappa_{\mathrm{L}} & - & - & - & - & - & \uparrow \\ \rho_{\mathrm{L}} & - & - & - & - & - & \downarrow \\ X_{\mathrm{spMM}} & - & - & \downarrow & - & - & \uparrow \\ f_{\mathrm{XspMM}} & \downarrow & \downarrow & - & - & \downarrow & \uparrow\end{array}$

${ }^{\mathrm{a}} \kappa_{\mathrm{TL}}<\kappa_{\mathrm{i}}=\kappa_{\mathrm{a}}$.

${ }^{\mathrm{b}} \kappa_{\mathrm{TL}}>\kappa_{\mathrm{i}}=\kappa_{\mathrm{a}}$.

As shown in Table 1, the effects of the changes in the structure of the T-tubules were dependent on direction; the effects in the transversal direction were more significant than those in the longitudinal direction. In some cases, the changes in the structure of the T-tubule caused opposite results, i.e. the increase in $W_{\mathrm{T}}$ caused the increase in $f_{\mathrm{YLL}}$ in the transversal direction, however, it caused the decrease in $f_{\mathrm{YLL}}$ in the longitudinal direction, and under the condition that $\kappa_{\mathrm{TL}}<\kappa_{\mathrm{a}}=\kappa_{\mathrm{i}}$, the increase in $\kappa_{\mathrm{TL}}$ caused the increase in $X_{\mathrm{spMM}}$ in the transversal direction, however, it caused the decrease in $X_{\mathrm{spMM}}$ in the longitudinal direction.

\subsection{Comparison with experimental results}

3.3.1. Comparison with the observed frequency-dependence of $\varepsilon, \kappa, \rho$, and $X_{s p}$. In the skeletal muscle excised from a dog [15], $\varepsilon$ at $20 \mathrm{~Hz}$ in the transversal and longitudinal directions was found to be $10^{6}$ and $10^{7}$, respectively. In the theoretical results shown in figure $16, \varepsilon$ in both directions at the low-frequency limit is comparable to the observed values. This suggests that the electrically inhomogeneous structure created by the existence of the T-tubules is enough to explain the large value of $\varepsilon$ observed at low frequencies. 
However, there were differences between the experimental results obtained from a previous study [15] and the theoretical ones shown in figure 16. To begin with, in the transversal direction below $100 \mathrm{~Hz}, \varepsilon$ continued to increase as the frequency decreased in the experimental results; however, $\varepsilon$ was found to be independent of frequency in the theoretical results. Presumably, $\varepsilon$ in the experimental results was dependent on frequency because the muscle cells of various diameters were included in the specimen. Secondly, in the longitudinal direction, the frequency-dependence of the experimental values of $\rho$ and $X_{\mathrm{sp}}$ were found between $10 \mathrm{kHz}$ and $1 \mathrm{MHz}$. Furthermore, in another experimental study that used specimen excised from wild-type and mdx mice [4], the frequency-dependence of $\rho$ in the longitudinal direction was observed in this region. The frequency-region in the experimental results was significantly higher than that found by the theoretical results (which was 1-100 Hz). The reason for this difference kind is not clear at this stage. One potential reason may be that long axis of the muscle cells deviated from being parallel to the external electric field, which was a condition assumed in the present study. The calculations made using modified models are required for examining the effects of the angle between the long axis of a cell and an external filed, and we plan to study them in the future.

3.3.2. Effects of muscle contractions. Recent observations of the muscles of mice in a transversal direction between $2 \mathrm{kHz}$ and $1 \mathrm{MHz}$ have demonstrated that muscle contractions caused increases in $R$ below $4 \mathrm{kHz}$ and decreases in the frequency at which $X$ was minimum [1].

The muscle contractions cause several changes to occur in the morphology and electrical properties of muscle cells, such as decreasing the distance between T-tubule networks [10], folding of surface membranes [11], and the increasing the trans-membrane capacitance [12, 13]. Because the volume of the muscle cells is expected to not change despite the contractions, the decrease in the length of muscle cells due to contractions causes cell diameters to increase. FDM calculations using $\kappa_{\text {SM-eq-T }}^{*}$ and $\kappa_{\text {SM-eq-L }}^{*}$ were carried out in order to examine the effects of these changes caused by the contractions. Table 2 shows the parameter-values that were used for the relaxed and contracted states of a muscle.

Table 2. Values of $L_{\mathrm{f}-\mathrm{xy}}, n_{\mathrm{T}}, \varepsilon_{\mathrm{SM}}$ of surface membranes used in calculations using $\kappa_{\mathrm{SM} \text {-eq-T }}^{*}$ and $\kappa_{\mathrm{SM}-\mathrm{eq}-\mathrm{L}}^{*}$ for the relaxed and contracted states of a muscle.

\begin{tabular}{lll} 
& Relaxed & Contracted \\
\hline$L_{\mathrm{f}-\mathrm{xy}} / \mu \mathrm{m}$ & 100 & 141 \\
$n_{\mathrm{T}} / \mu \mathrm{m}^{2}$ & 0.495 & 0.700 \\
$\varepsilon_{\mathrm{SM}}$ & 10.0 & 14.2
\end{tabular}

Similarly to the examinations described in 2.1.4., the widths of the I band in the relaxed and contracted states were made to be $2.4 \mu \mathrm{m}$ and $0.4 \mu \mathrm{m}$, respectively. The width of the A band was made to be $1.6 \mu \mathrm{m}$ and remained unchanged. As a result, the lengths sarcomeres in the relaxed and contracted states were $4.0 \mu \mathrm{m}$ and $2.0 \mu \mathrm{m}$, respectively. T-tubule networks were placed at the junction between the I and A bands.

In table $2, L_{\mathrm{f}-\mathrm{xy}}$ and $\varepsilon_{\mathrm{SM}}$ in the relaxed state were made to be $100 \mu \mathrm{m}$ and 10 , respectively; these were the same values as the initial values used in 2.1.2.. $L_{\mathrm{f}-\mathrm{xy}}$ in the contracted state was evaluated so that the cell volume remained unchanged. As a result, the decrease in the length of sarcomeres from $4.0 \mu \mathrm{m}$ to $2.0 \mu \mathrm{m}$ due to the contractions caused $L_{\mathrm{f}-\mathrm{xy}}$ to increase from $100 \mu \mathrm{m}$ to $141 \mu \mathrm{m}$. The changes due to the contractions caused the area of the hypothetical smooth membrane to decrease in each sarcomere. This caused $n_{\mathrm{T}}$ to increase, as shown in table 2. Because the area of the surface membrane 
within the cell was assumed to not change, the decrease in the area of the hypothetical smooth membrane in each sarcomere was expected to cause the surface membranes to fold. This effect was represented by the increased value of $\varepsilon_{S M}$ in the contracted state, which can be seen in table 2 . In both the relaxed and the contracted states, $\varepsilon_{\mathrm{TM}}$ was 10 . In addition, because both the number of T-tubules contained in each T-tubule network and $W_{\mathrm{T}}$ were assumed to not change, the capacitance due to the tubular membrane increased when $L_{\mathrm{f}-\mathrm{xy}}$ increased due to the contraction by a factor of 1.4 , which was consistent with the experimental results $[12,13]$.

Figure 17 shows $\varepsilon, \kappa, \rho$, and $X_{\mathrm{sp}}$ obtained using the FDM calculations for the models similar to those shown in figures 3 and 4 ; these models had smooth surface membranes characterized by $\kappa_{\text {SM-eq-T }}^{*}$ and $\kappa_{\text {SM-eq-L }}^{*}$, which were evaluated using the values of the parameters shown in Table 2 , as well as the values described in 2.1.2. (namely, $\varepsilon_{\mathrm{i}}=\varepsilon_{\mathrm{a}}=\varepsilon_{\mathrm{TL}}=80, \kappa_{\mathrm{i}}=\kappa_{\mathrm{a}}=\kappa_{\mathrm{TL}}=2 \mathrm{~S} / \mathrm{m}, \varepsilon_{\mathrm{TM}}=10$, $\kappa_{\mathrm{SM}}=\kappa_{\mathrm{TM}}=0, T_{\mathrm{SM}}=T_{\mathrm{TM}}=7 \mathrm{~nm}$ and $\left.T_{\mathrm{o}-\mathrm{xy}}=5 \mu \mathrm{m}\right) . L_{\mathrm{f}-\mathrm{z}}$ in these models for the transversal and longitudinal directions was set to $1 \mu \mathrm{m}$ and $1 \mathrm{~mm}$, respectively.

The changes in $\rho$ and $X_{\mathrm{sp}}$ in the transversal direction (figure 17 (b)) due to the muscle contractions were in good agreement with those in $R$ and $X$ in the transversal direction that have previously been obtained experimentally [1]. Compared with the results of previous theoretical examinations made using models without T-tubules [9], the effects of the contractions on $\varepsilon, \kappa, \rho$, and $X_{\mathrm{sp}}$ in the transversal direction were the same qualitatively as those caused by the increase in $L_{\mathrm{f} \text {-xy }}$. However, in the longitudinal direction, the effects of the contractions were different from those caused by the increase in $L_{\mathrm{f}-\mathrm{xy}}$; in the case of the models that did not contain T-tubules, the increase in $L_{\mathrm{f}-\mathrm{xy}}$ caused $\varepsilon$ to decrease in the low-frequency limit, $\rho$ to increase in around $f_{\mathrm{XspMM}}$, and $X_{\mathrm{sp}}$ to decrease at frequencies higher than $f_{\mathrm{XspMM}}$. The increase in $L_{\mathrm{f}-\mathrm{xy}}$ did not cause $f_{\mathrm{XspMM}}$ to change. These changes were different from those shown in figure 17. Unfortunately, the validity of the theoretical predictions cannot currently be assessed due to the disagreement between the theoretical and experimental results in the longitudinal direction, which is described in detail in 3.3.1..
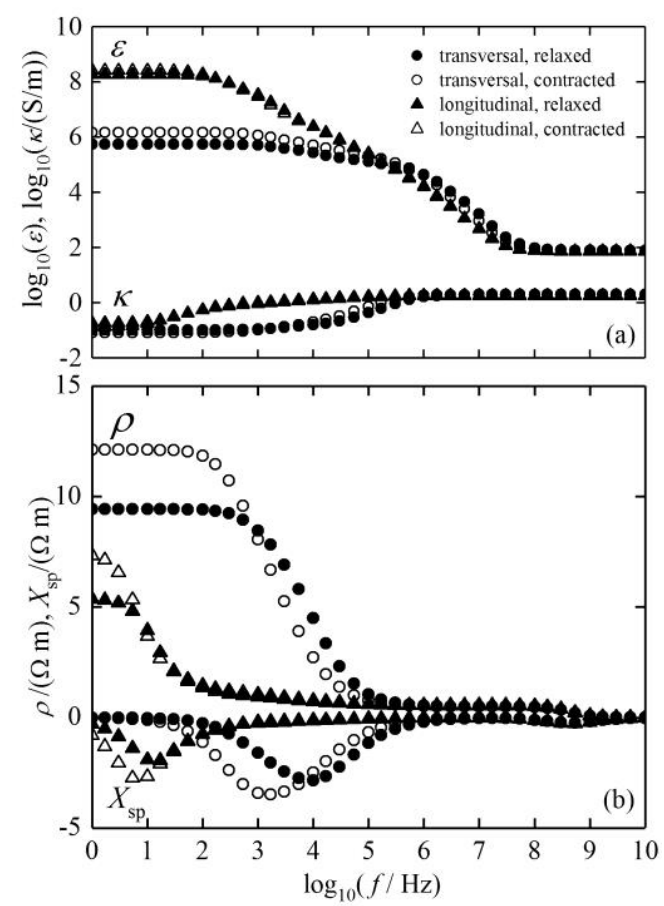

Figure 17. Effects of muscle contractions on the frequency-dependence of $\varepsilon, \kappa, \rho$, and $X_{\mathrm{sp}}$ in the transversal and longitudinal directions as obtained using models that are large enough to be comparable to real muscle cells. The difference between the relaxed and contracted states was characterized by the parameter-values shown in table 2 . 


\section{Conclusion}

As described in 2.2. and 3.1.1., the present study demonstrated that the effects of the T-tubules were different in the transversal and longitudinal directions, which was a finding that was contrary to that found by a previous study [17]. This is the first case in which the direction-dependence of the effects of T-tubules has been shown.

In the process of deriving $\kappa_{\mathrm{SM} \text {-eq-T }}^{*}$, the angle between surface membranes and an external electric field, $\mathbf{E}_{\mathrm{ex}}$, and that between a tubule and $\mathbf{E}_{\mathrm{ex}}$ were restricted to being either 0 degree or 90 degrees. Irrespective of this assumption, $\kappa_{\mathrm{SM} \text {-eq-T }}^{*}$ was successful in cases in which the angles deviated from the assumed ones, as described in 3.1.6.. Additionally, the analytical relations for $\kappa_{\mathrm{SM} \text {-eq-T }}^{*}$ and $\kappa_{\mathrm{SM} \text {-eq-L }}^{*}$ do not contain parameters unique to the assumed structures of the models used in the derivation. From this finding, it is expected that $\kappa_{\mathrm{SM} \text {-eq-T }}^{*}$ and $\kappa_{\mathrm{SM} \text {-eq-L }}^{*}$ can be used to represent the effects of T-tubules in real muscle cells whose structures deviate from those of the models used in the derivation; at the very least, they can be used as approximated relations in the initial stages of examinations.

We determined that using equivalent complex conductivities allowed for the effects of T-tubules and folded surface membranes to be examined using the results of calculations made using cell models that were large enough in size to be comparable to real muscle cells, as described in 3.2. and 3.3.. Calculations such as these are expected to increase the knowledge about the effects of structural changes of muscles on their $Y$ and $Z$. The knowledge, which has been collected in parallel with histological and physiological studies, is expected to contribute to the development of the structural and functional analyses of the skeletal muscles made using measurements of $Y$ and $Z$.

\section{Acknowledgements}

I thank Dr. T. Yamazaki for helpful advice about the changes in the muscle structure due to the contraction and A. Yamada, H. Kageyama, T. Igarashi, and N. Yamamoto who worked on this subject at preliminary stages, as a part of the undergraduate program. This research was supported by JSPS KAKENHI (Grant no. 16K12928).

\section{Appendix A. Procedure used to derive the analytical relations used for the equivalent complex conductivity}

The theoretical relation for the admittance, $Y_{\mathrm{T}}$, due to a single tubule was derived using cable-line theory [19] by assuming that a tubule was straight and did not have branches, and that $\phi=0$ inside a cell. Under this condition, the current, $I_{\mathrm{TL}}$, and the potential, $\phi_{\mathrm{TL}}$, in a tubular lumen can be represented as (A.1) and (A.2) as functions of the position $u$ corresponding to $x$ and $y$ in the cell models shown in figures 3 and 4 .

$$
\begin{aligned}
& I_{\mathrm{TL}}=c_{1} \exp (\gamma u)+c_{2} \exp (-\gamma), \\
& \phi_{\mathrm{TL}}=-\sqrt{\frac{Z_{\mathrm{TL}}}{Y_{\mathrm{TM}}}}\left[c_{1} \exp (\gamma u)-c_{2} \exp (-\gamma)\right],
\end{aligned}
$$

where $c_{1}$ and $c_{2}$ are constants that are determined using the boundary conditions with respect to $I_{\mathrm{TL}}$ and $\phi_{\mathrm{TL}}$, and $\gamma=\sqrt{Y_{\mathrm{TM}} Z_{\mathrm{TL}}}$, where $Y_{\mathrm{TM}}$ and $Z_{\mathrm{TL}}$ are values of the impedance of the tubular lumen and the admittance of the tubular membrane, respectively, in a unit length of a tubule. In order to derive the relations for $Y_{\mathrm{T}}$, the tubules in the models were classified into two groups as shown in figure 8; T-PL represents a tubule parallel to $\mathbf{E}_{\mathrm{ex}}$, and T-PP represents a tubule perpendicular to $\mathbf{E}_{\mathrm{ex}}$. According to the 
symmetry of the shape of Model-T and Model-L shown in figures 3 and 4, respectively, $\phi_{\mathrm{TL}}=0$ at $u=$ 0 in T-PL, and $I_{\mathrm{TL}}=0$ at $u=0$ in T-PP. Using these relations, $c_{1}$ and $c_{2}$ in (A.1) and (A.2) for T-PL and T-PP can be determined. By assuming $Y_{\mathrm{T}}=-I_{\mathrm{TL}} / \phi_{\mathrm{TL}}$ using $I_{\mathrm{TL}}$ and $\phi_{\mathrm{TL}}$ at the surface of a cell where $u=L_{\mathrm{f}-\mathrm{xy}} / 2$, the admittance of T-PP, $Y_{\mathrm{T}-\mathrm{PP}}$, and that of T-PL, $Y_{\mathrm{T}-\mathrm{PL}}$, can be obtained as (3) and (4) in the text, respectively.

The relations for the equivalent complex conductivity of a hypothetical smooth surface membrane were derived using the admittance of a cell surface, $Y_{\mathrm{CS}}$. In the case of Model-T, shown in figures 8(T-1) and 8(T-2), two kinds of $Y_{\mathrm{CS}}$ needed to be considered: that of the cell surface parallel to $\mathbf{E}_{\text {ex }}, Y_{\text {CS-PL }}$, and that of the one perpendicular to $\mathbf{E}_{\text {ex }}, Y_{\text {CS-PP. }}$ These were represented as summations of the admittance of the surface membrane and the tubules:

$$
\begin{aligned}
& Y_{\mathrm{CS}-\mathrm{PL}}=\kappa_{\mathrm{SM}}^{*} S_{\mathrm{SM}-\mathrm{PL}} / T_{\mathrm{m}}+N_{\mathrm{T}-\mathrm{PP}} Y_{\mathrm{T}-\mathrm{PP}}, \\
& Y_{\mathrm{CS}-\mathrm{PP}}=\kappa_{\mathrm{SM}}^{*} S_{\mathrm{SM}-\mathrm{PP}} / T_{\mathrm{m}}+N_{\mathrm{T}-\mathrm{PL}} Y_{\mathrm{T}-\mathrm{PL}},
\end{aligned}
$$

where $S_{\mathrm{SM}-\mathrm{PL}}$ and $N_{\text {T-PP }}$ are the area of a surface membrane and the number of mouths of the tubules, respectively, included for the surface CS-PL, while $S_{\mathrm{SM}-\mathrm{PP}}$ and $N_{\mathrm{T}-\mathrm{PL}}$ are those parameters for CS-PP. The $S_{\mathrm{SM}-\mathrm{PL}}$ and $S_{\mathrm{SM}-\mathrm{PP}}$ parameters were increased by the folding of a surface membrane.

Our previous study [9] showed that, in the transversal direction, a summation of one-third of the capacitance of a surface membrane at CS-PL and all of the capacitance of a surface membrane at CS-PP contributed to the permittivity of muscles at the low-frequency limit under the condition that $\kappa_{\mathrm{SM}}=0$. From this result, we expected that $Y$ and $Z$ of muscles in the transversal direction would be affected by $Y_{\mathrm{CS}-\mathrm{PL}} / 3+Y_{\mathrm{CS}-\mathrm{PP}}$. This must be equal to an admittance in which both $Y_{\mathrm{CS}-\mathrm{PL}}$ and $Y_{\mathrm{CS}-\mathrm{PP}}$ are represented using the equivalent complex conductivity $\kappa_{\mathrm{SM}-\mathrm{eq}-\mathrm{T}}^{*}$ for the transversal direction:

$$
\kappa_{\mathrm{SM}-\mathrm{eq}-\mathrm{T}}^{*}\left(S_{\mathrm{SM}-\mathrm{PL}-0} / 3+S_{\mathrm{SM}-\mathrm{PP}-0}\right) / T_{\mathrm{m}}=Y_{\mathrm{CS}-\mathrm{PL}} / 3+Y_{\mathrm{CS}-\mathrm{PP}},
$$

where $S_{\mathrm{SM}-\mathrm{PL}-0}$ and $S_{\mathrm{SM}-\mathrm{PP}-0}$ are the areas of hypothetical smooth surface membranes at CS-PL and CS-PP, respectively. In the Model-T shown in figure 3, $S_{\mathrm{SM}-\mathrm{PL}}=S_{\mathrm{SM}-\mathrm{PP}}=S_{\mathrm{SM}} / 2, N_{\mathrm{T}-\mathrm{PL}}=N_{\mathrm{T}-\mathrm{PP}}=N_{\mathrm{T}} / 2$ and $S_{\mathrm{SM}-\mathrm{PL}-0}=S_{\mathrm{SM}-\mathrm{PP}-0}=S_{\mathrm{SM}-0} / 2$, where $S_{\mathrm{SM}}$ is the area of a surface membrane, $N_{\mathrm{T}}$ is the number of mouths of the tubules included in the realistic model, and $S_{\mathrm{SM}-0}$ is the area of a hypothetical smooth surface membrane. From (A.1) to (A.5), we obtained (1) in the text for Model-T. As shown in figures 8(L-1) and 8(L-2), Model-L consists of T-PP and CS-PL; as a result, the equivalent complex conductivity $\kappa_{\mathrm{SM}-\mathrm{eq}-\mathrm{L}}^{*}$ for Model-L can be represented by (2).

\section{References}

[1] Sanchez B, Li J, Geisbush T, Bardia R B and Rutkove S B 2016 Impedance alterations in healthy and diseased mice during electrically induced muscle contraction IEEE Trans. Biomed. Eng. 63 $1602-12$

[2] Shiffman C A 2016 Pre-contraction dynamic electrical impedance myography of the forearm finger flexors Physiol. Meas. 37 291-313

[3] Nakamura T, Kusuhara T and Yamamoto Y 2013 Motion discrimination of throwing a baseball using forearm electrical impedance J. Phys. Conf. Ser. 434012070

[4] Rutkove S B et al. 2016 Loss of electrical anisotropy is an unrecognized feature of dystrophic muscle that may serve as a convenient index of disease status Clinical Neurophysiol. 1273546 551

[5] Benatar M, Boylan K, Jeromin A, Rutkove S B, Berry J, Atassi N and Bruijn L 2016 ALS biomarkers for therapy development: state of the field and future directions Muscle Nerve $\mathbf{5 3}$ $169-82$ 
[6] Rutkove S B 2015 Clinical Measures of Disease Progression in Amyotrophic Lateral Sclerosis Neurotherapeutics 12 384-93

[7] Zaidman C M et al 2015 Electrical impedance myography in duchenne muscular dystrophy and healthy controls: a multicenter study of reliability and validity Muscle Nerve 52 592-7

[8] Li J, Geisbush T R, Arnold W D, Rosen G D, Zaworski P G and Rutkove S B 2014 A comparison of three electrophysiological methods for the assessment of disease status in a mild spinal muscular atrophy mouse model PLOS One 9 e 111428

[9] Sekine K 2015 Numerical calculations for effects of structure of skeletal muscle on frequency-dependence of its electrical admittance and impedance J. Phys. D: Appl. Phys. $\mathbf{4 8}$ 255401

[10] Ross M H and Pawlina W 2006 Histology: A Text and Atlas (Philadelphia: Lippincott Williams \& Wilkins)

[11] Dulhunty A F and Franzini-Armstrong C 1975 The relative contributions of the fold and caveolae to the surface membrane of frog skeletal muscle fibres at different sarcomere lengths $J$. Physiol. $250513-39$

[12] Takashima S 1985 Passive electrical properties and voltage dependent membrane capacitance of single skeletal muscle fibers Pflugers Arch. 403 197-204

[13] Takashima S 1989 Electrical Properties of Biopolymers and Membranes (Philadelphia: Adam Hilger)

[14] Eisenberg R S 1983 Impedance measurement of the electrical structure of skeletal muscle Handbook of Physiology ed L D Peachey et al (Bethesda: American Physiological Society) pp 301-23 (sect 10)

[15] Epstein B R and Foster K R 1983 Anisotropy in the dielectric properties of skeletal muscle Med. Biol. Eng. Comput. 21 51-5

[16] Foster K R and Schwan H P 1996 Dielectric properties of tissues Handbook of Biological Effects of Electromagnetic Fields 2nd edn, ed C Polk and E Postow (Boca Raton, FL: CRC Press) pp 25102

[17] Roth B J, Gielen F L H and Wikswo Jr J P 1988 Spatial and temporal frequency dependent conductivities in volume-conduction calculations for skeletal muscle Math. Biosci. 88 159-89

[18] Schwan H P 1963 Determination of biological impedance Physical Tchniques in Biological Research, volume 6 part B, ed WL Nastuk (New York: Academic Press) pp 323-407

[19] Stratton J A 1941 Electromagnetic Theory (New York: McGraw-Hill)

[20] Eisenberg B R 1983 Quantitative ultrastructure of mammalian skeletal muscle Handbook of Physiology ed L D Peachey et al (Bethesda: American Physiological Society) pp 73-112 (sect 10)

[21] Asami K 2006 Dielectric dispersion in biological cells of complex geometry simulated by the three-dimensional finite difference method J. Phys. D: Appl. Phys. $39492-99$ 